\title{
Single-leg weight limit of fixation model of simple supracondylar fracture of femur
}

\author{
Guo-Yu Bai ${ }^{1,2} \cdot$ Xiang-Hong Xü ${ }^{2}$ Jin-Hui Wang ${ }^{3} \cdot$ Ning Sun ${ }^{3}$
}

Received: 6 December 2018 / Revised: 4 January 2019 / Accepted: 10 January 2019 / Published online: 22 May 2019

(C) The Chinese Society of Theoretical and Applied Mechanics and Springer-Verlag GmbH Germany, part of Springer Nature 2019

\begin{abstract}
Early postoperative rehabilitation training for supracondylar fracture of femur aids in accelerating healing with shorter recovery periods. Presently, clinical studies on early postoperative weight training are still in the nascent stage. The weight-bearing capacity at different healing stages typically depends on clinical experience, and there is a lack of standards to quantify the weight that is conducive to healing of fractures. In this paper, a three-dimensional (3D) geometric model of the femur is obtained using imaging data, a locking plate fixation model of a simple supracondylar fracture of the femur, considering the angle and spatial direction of the fracture surface, is established, the stress distribution and load transmission mechanism of the fracture fixation model in a single-leg standing posture are studied, and the weight-bearing capacity of a standing single leg at the early stage of fracture is given. This provides the basis for objective quantification of early postoperative weight-bearing capacity.
\end{abstract}

Keywords Simple supracondylar fracture of femur $\cdot$ Early weight bearing $\cdot$ Single-leg weight limit

\section{Introduction}

Distal femoral fractures account for $4 \%-7 \%$ of all femoral fractures and $0.4 \%$ of systemic fracture dislocations [1]. According to the Arbeitsgemeinschaft für Osteosynthesefragen (AO) classification principle, type A distal femoral fractures [2], that is, supracondylar fractures of the femur, are unstable and can easily spread to the articular surface, with many complications and a high disability rate, therefore accounting for one of the most difficult fractures to treat. Moreover, as the outcomes of conservative treatment are poor, active surgical treatment is advised at present. Supracondylar fractures of the femur, especially comminuted or osteoporotic fractures, are usually treated with locking plates [3]. The advantage of this approach is that the incision is

Xiang-Hong Xu

xxh@lnm.imech.ac.cn

$1 \quad$ Tianjin Key Laboratory of Modern Engineering Mechanics, Department of Mechanics, Tianjin University, Tianjin 300072, China

2 State Key Laboratory of Nonlinear Mechanics, Institute of Mechanics, Chinese Academy of Sciences, Beijing 100190, China

3 Beijing Jishuitan Hospital, Beijing 100035, China small, hence can be considered minimally invasive, and the ligament and nerves around the knee joint are not affected greatly, which is beneficial to the function of the knee joint $[4,5]$. On the one hand, a gap between the locking plates and the bone is maintained, which mitigates any adverse effect on the blood flow over the bone surface under the plate; on the other hand, locking plate fixation is a type of bridge fixation that allows the fracture site to be moved slightly, thereby facilitating fracture healing $[6,7]$.

Fracture healing after locking plate fixation is characterized as secondary healing [2]. The callus is renewed, and its formation can be categorized into various stages, namely the inflammatory stage, soft callus formation stage, hard callus formation stage, and remodeling stage. Therefore, the material properties and bearing capacity of the callus are different in different healing stages.

In fact, stress or strain stimulation can promote bone tissue growth, and factors such as the magnitude, direction, and timing of stress may affect fracture healing, providing additional insight into and a theoretical basis for early postoperative rehabilitation treatment [8-10]. Using experiments on sheep tibia, Kenwright et al. [11] showed that a fracture with slight movement at the fracture site formed mature bridge calluses after 12 weeks, while for a fracture without stimulation, no complete callus formed. In the work of 
Goodship et al. [12], stimulation of sheep tibia was begun in two groups at 1 and 6 weeks after operation, revealing that, in the group with earlier stimulation, the bone mineral density in the healing area increased at a higher rate.

Current clinical attempts at early weight training provide strong evidence that early rehabilitation treatment can reduce healing time. Locking plate fixation was used by Liang et al. [4] for distal femur fractures. The patients performed functional exercises on the second day after the operation, and the callus formed normally without any complications or failure of the locking plate. Intramedullary nail fixation was used by Arazi et al. [13] for femoral shaft comminuted fracture, and the patients performed weight training 1 week after the operation and could carry weight completely 2 months after the operation without the aid of assistive devices. The patients all ultimately recovered. Minimally invasive locking plate fixation was used by Adam et al. [14] for tibial shaft fracture, and the patients performed weight training directly after the operation. Healing took an average of about 9.1 weeks. Open reduction and internal fixation for pelvic ring fracture was used by Kalmet et al. [15], and the patients underwent weight training 10 days after the operation. They could walk independently and even run within 7 weeks.

However, such clinical study on early postoperative weight training is still in a nascent stage. The weight capacity at different healing stages mainly depends on clinical experience, and there is still no quantitative weight standard that can be considered conducive to fracture healing. Scholars have carried out extensive research in this area. By taking the strain magnitude of internal fragments as baseline parameters, Claes [6] theoretically analyzed the required stiffness of a fixed structure as a function of the transverse fracture gap, revealing that, as the fracture gap is varied, the effect of fracture healing can be improved by changing the stiffness of the fixed structure. In their work, Elkins et al. [16] found that, the greater the distance between screws, the greater the transverse displacement of the transverse fracture site, being unfavorable for fracture healing. The results of animal experiments by Bottlang et al. [17] showed that symmetric and uniform axial motion promotes consistent and circumferential bridging, delivering faster callus formation.

Clinical cases of simple supracondylar fracture of the femur are complicated and diverse, the angle and spatial direction of the fracture surface are randomly uncertain, and transverse fracture is only one of them. A fixation model for simple supracondylar fractures of the femur considering the angle and spatial direction of the fracture surface is established herein, the stress characteristics and transmission mechanism of each part of the fixation model are studied, and the ultimate weight capacity of the fracture fixation model at different healing stages is given, providing a theoretical basis for early rehabilitation training of supracondylar fracture of the femur.

\section{Materials and methods}

\subsection{Geometry modeling}

Figure 1a shows quantitative computed tomography (QCT) images in the right coronal plane of the femur in adults. A Toshiba spiral computed tomography (CT) was used, with scanning parameters of $120 \mathrm{kVp}$ (kilovolt peak) and 150 mAs (milliampere second) and layer thickness of $1 \mathrm{~mm}$. The 3D geometric model of the adult right femur was obtained by 3D reconstruction of QCT scanning data (Fig. 1b). The cross-section of the supracondylar transverse fracture of the femur was perpendicular to the anatomical axis, $65 \mathrm{~mm}$ from the lower end of the femur, and the fracture gap was $3 \mathrm{~mm}$, in which healing tissue was filled.

Clinically, the metal locking plate system produced by Zimmer was used for fixation (Fig. 1c, d). Specifically, a 10/14 lateral hole locking plate for a right distal femur with length of 223/286 mm was chosen; a total of nine screws were arranged on two plates, including five hollow $4.5-\mathrm{mm}$ lock screws (internal and external diameter of $3.43 \mathrm{~mm}$ and $4.50 \mathrm{~mm}$ ) in the femoral shaft and four hollow $5.5-\mathrm{mm}$ lock screws (internal and external diameter of $4.75 \mathrm{~mm}$ and $5.51 \mathrm{~mm}$ ) in the femoral condyle. In the modeling, the screw direction is chosen perpendicular to the locking plate. More specifically, the screw direction is perpendicular to the anatomical axis of the femur in the straight area of the plate and perpendicular to the mechanical axis of the femur at the femoral condyle. The screw is simplified as a cylinder, where the diameter of the cylinder is the outer diameter of the screw, and the diameter of the screw hole is the outer diameter of the screw. The depth of the screw threads is considered negligible and hence ignored (Fig. 1e).

The angle between the cross-section of a simple supracondylar fracture of the femur and the anatomical axis can vary. Figure 2 shows four typical clinical cases with angle of $0^{\circ}, 30^{\circ}, 45^{\circ}$, and $60^{\circ}$, respectively, and a fracture gap of $3 \mathrm{~mm}$. Specifically, transverse fractures and oblique fractures at $30^{\circ}$ and $45^{\circ}$ are fixed with a 10-hole lateral locking plate of the distal femur; the fracture surface of oblique fractures at $60^{\circ}$ is longer along the femoral axis, hence the screw cannot be fixed on the fracture surface, and the fractures are fixed with a 14-hole locking plate. The cross-section of the fractures is located in the middle of the adjacent screws. The upper end of the cross-section of transverse fractures and oblique fractures at $60^{\circ}$ are $65 \mathrm{~mm}$ and $138 \mathrm{~mm}$ from the lower end of the femur, respectively, with a gap of $60-150 \mathrm{~mm}$ between the fracture and locking plate.

Further clinical statistics show that, even when the angle between the fracture cross-section and anatomical axial cross-section is found, there may be many different spatial directions of the fracture cross-section. Figure 3 shows the 

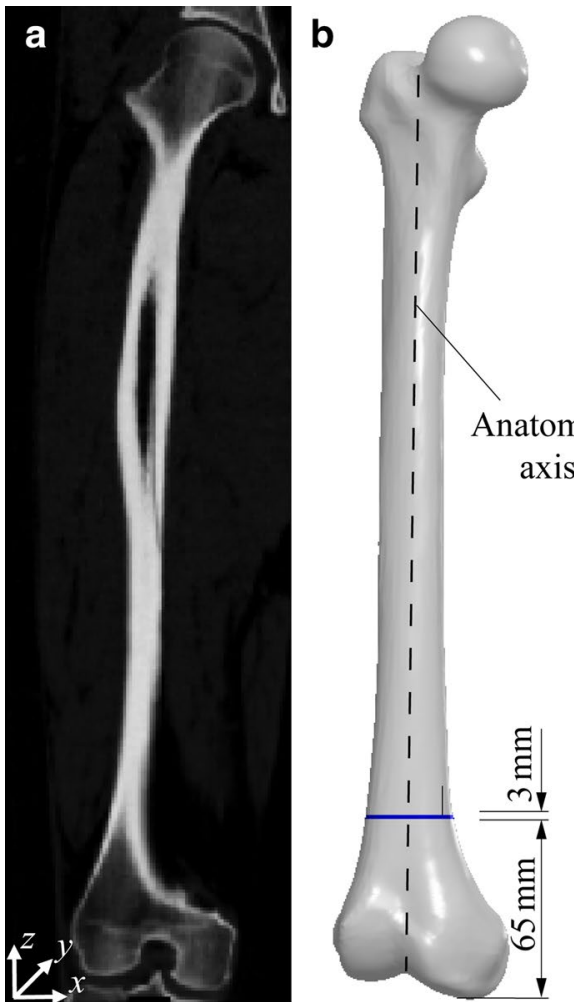

C

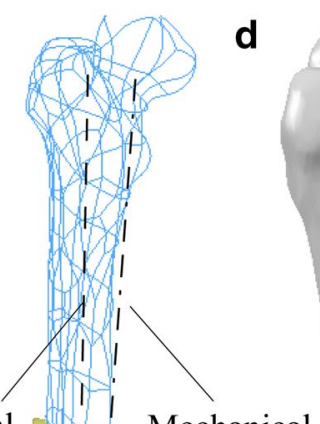
axis

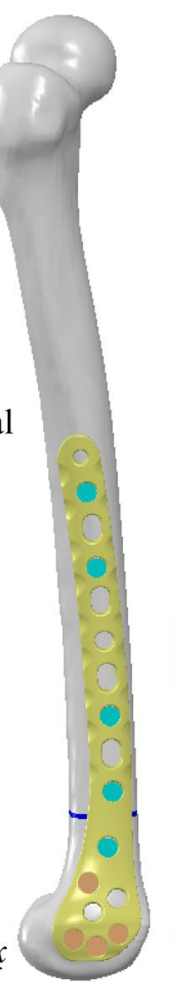

e

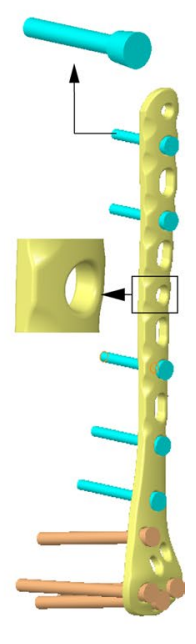

Fig. 1 Fixation model of simple supracondylar fracture of femur: a QCT image of right coronal plane of femur; $\mathbf{b}$ 3D geometry model; $\mathbf{c}, \mathbf{d}$ surgical fixation model; e simplified three-dimensional geometry model of locking plate and screw produced by Zimmer. The magnified figures of the screw and screw hole show that the screw threads were ignored and simplified as cylindrical surfaces

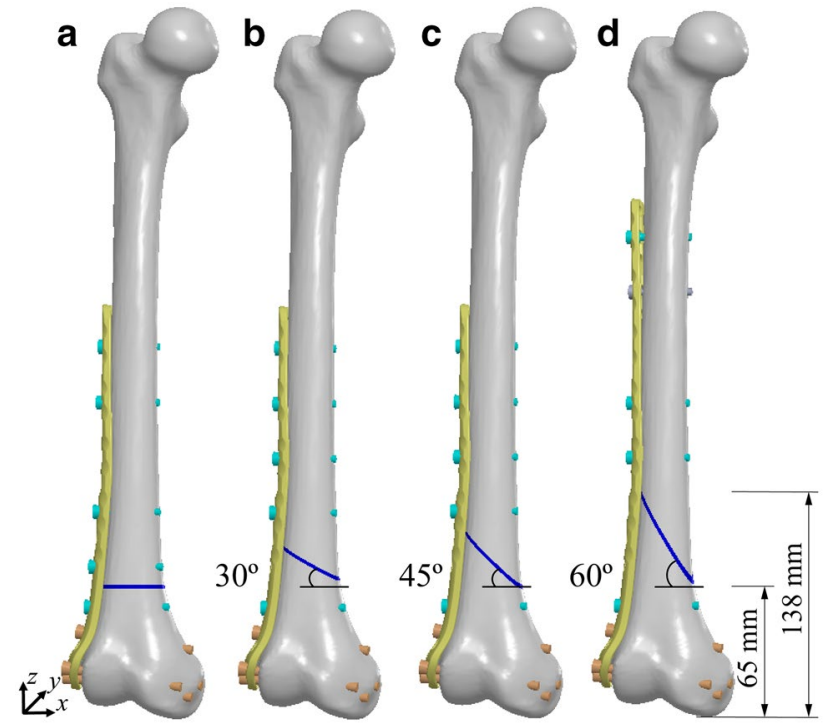

Fig. 2 Fixation scheme of simple fracture. The angle between the fracture cross-section and anatomical axis cross-section is $\mathbf{a} 0^{\circ}, \mathbf{b} 30^{\circ}$, c $45^{\circ}$, and $\mathbf{d} 60^{\circ}$ where a 10 - or 14 -hole locking plate is used. The fracture cross-section is located in the middle of the adjacent screws

four typical spatial directions of the fracture surface. For direction I, the fracture surface is perpendicular to the frontal plane, and the fracture site on the side of the plate is higher than the other side of the plate; For direction II, III, and IV, the fracture surface is rotated by $90^{\circ}, 180^{\circ}$, and $270^{\circ}$ counterclockwise (quarter turns) around the anatomic axis, respectively.

\subsection{Material properties}

QCT data can give the CT value $H_{\mathrm{b}}$ at any point in the femur, from which the QCT density $\rho_{\mathrm{QCT}}$ of bone mineral can be calculated. Experimentally, the $\rho_{\mathrm{QCT}}-H_{\mathrm{b}}$ relation is first determined by baseline calibration. It is known that the $\rho_{\mathrm{QCT}}$ values of the five tubes of baseline calibration are $0,0.05$, $0.1,0.15$, and $0.2 \mathrm{~g} / \mathrm{cm}^{3}$, respectively, with $H_{\mathrm{b}}$ values of the measured baseline of $-69,-15,200,378$, and $514 \mathrm{HU}$ (hounsfield unit), respectively, revealing a linear relationship (Fig. 4) of

$\rho_{\mathrm{QCT}}=0.0003132 H_{\mathrm{b}}+0.03685$.

The $\rho_{\mathrm{QCT}}$ at any point in the femur can then be calculated using Eq. (1).

Some studies have shown that the axial elastic modulus $E$ of bone tissue follows a quantitative relationship [18-28] with $\rho_{\mathrm{QCT}}$ (Fig. 5). Among them, the quantitative relationship between the bone apparent density $\rho_{\text {app }}$ and $\rho_{\mathrm{QCT}}$ given by Lotz et al. $[18,19]$, and the quantitative relationship between 


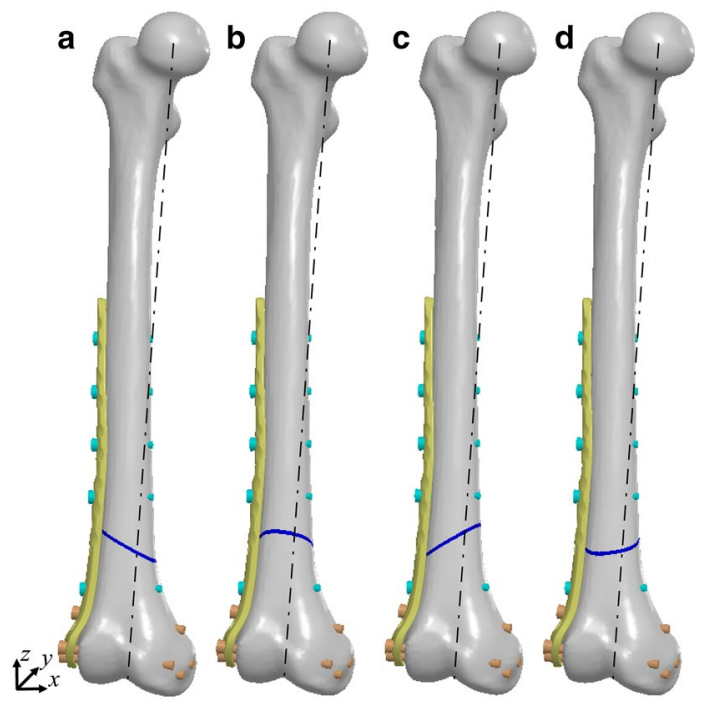

Fig. 3 Four typical spatial directions of fracture surface: a direction I, with the fracture surface perpendicular to the frontal plane ( $x z$ plane) and the lateral fracture end higher than the medial one. Directions b II, c III, and d IV are obtained when the fracture surface is rotated $90^{\circ}, 180^{\circ}$, and $270^{\circ}$ counterclockwise (quarter turn) around the anatomic axis, respectively

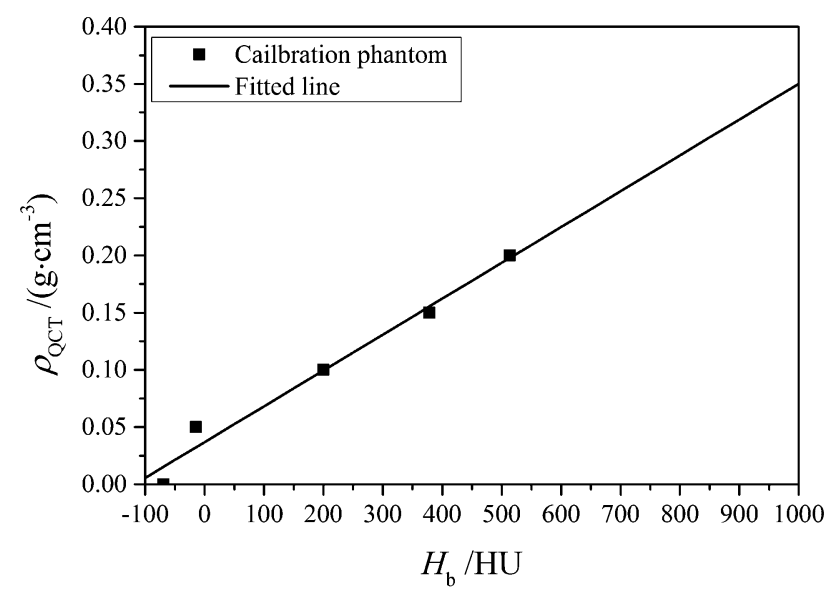

Fig. 4 Quantitative relation between bone mineral density $\rho_{\mathrm{QCT}}$ and CT value $H_{\mathrm{b}}$

bone ash density $\rho_{\text {ash }}$ and $\rho_{\mathrm{QCT}}$ given by Gong et al. [22, 23 ] and Keyak and Skinner [29], are transformed according to the empirical formulae $\rho_{\mathrm{QCT}}=\left(\rho_{\text {app }}-0.17\right) / 0.012$ and $\rho_{\mathrm{QCT}}=\left(\rho_{\text {ash }}-0.0457\right) / 0.000953$, respectively, but Carter and Hayes $[24,25]$ found that a power function is more suitable to describe the relationship between $E$ and $\rho_{\text {app }}$ than the linear function, and Bessho et al. [30] and Gong et al. [22] used a piecewise function to characterize the relationship between them. Because of the discreteness of biomaterials, there is a large scatter in the research results of many scholars. The $E$ values obtained for the same $\rho_{\mathrm{QCT}}$ in the

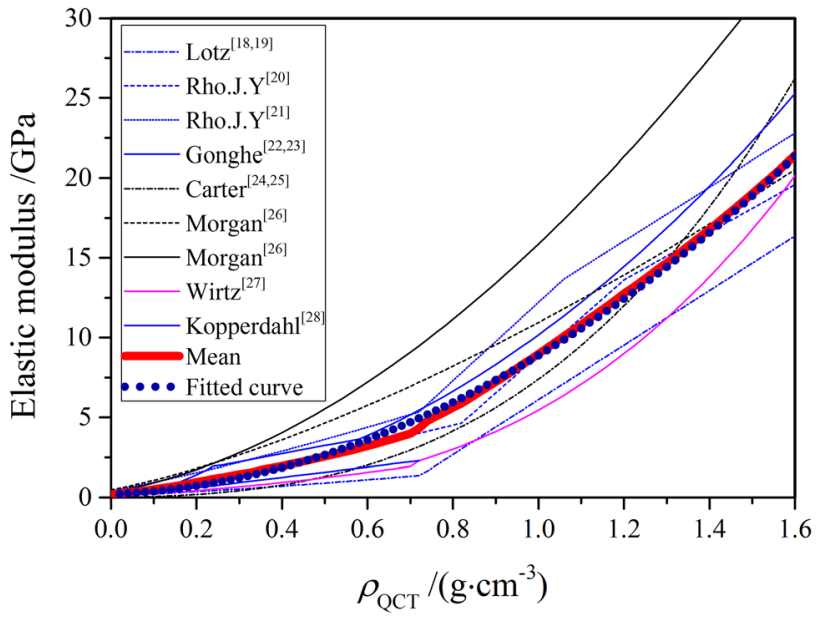

Fig. 5 Relationship between elastic modulus $E$ and bone mineral density $\rho_{\mathrm{QCT}}[18-28]$

above-cited literature reports were averaged in this paper (see thick solid line in Fig. 5), and the curve fitting results showed that $E$ and $\rho_{\mathrm{QCT}}$ satisfies a quadratic polynomial (Fig. 5, thick dashed line)

$E=7.553 \rho_{\mathrm{QCT}}^{2}+1.125 \rho_{\mathrm{QCT}}+0.2123$.

In this formula, the unit of $E$ is GPa. In this paper, the bone is treated as an isotropic linear elastic material with Poisson's ratio of 0.3 .

Bone tissue is heterogeneous, and there is a gradual transition between compact bone and cancellous bone rather than a strict boundary. To describe the nonuniform mechanical properties of bone, it is necessary to classify its elastic modulus, and the higher the grading number (that is the variation gradient of material properties), the closer it is to the actual structure. However, considering the scanning accuracy of CT and comprehensive finite element calculations, the optimal grading number of elastic modulus satisfying the calculation accuracy of the fracture model can be determined. According to 256 grayscale grades, Garcia et al. [31] divided the elastic modulus of bone into 256 grades, while Keyak et al. [32] took 5\% as an incremental step to divide the elastic modulus of bone into 170 grades, Dalstra et al. [33] suggested that the grading number of the elastic modulus of bone should be over 214, and Perillo-Marcone [34] divided the elastic modulus into over 500 grades. Figure 6 a shows the distribution of the elastic modulus of two typical sections of femoral shaft and femoral condyle when the grading number is 64 , and Fig. $6 \mathrm{~b}$ shows the distribution curve of the elastic modulus in the region with larger gradient changes for grading numbers of 2, 4, 8, 16, 32, 64, 128, and 256, respectively. It can be seen that, when the grading 

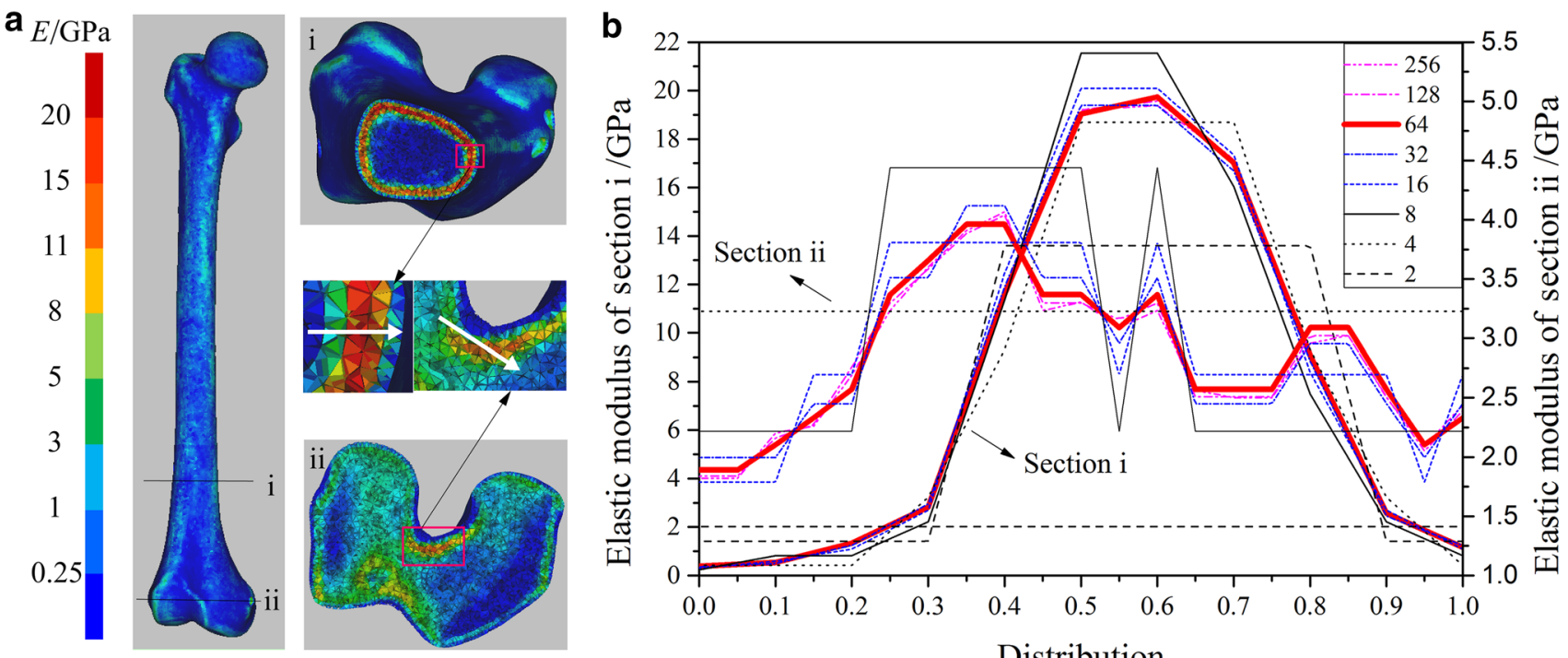

Distribution

Fig. 6 Discrete grading of the elastic modulus of right femur. a Grading number is 64 in sections of femoral shaft $i$ and femoral condyle ii. b Distribution curve of the elastic modulus in the region of the white arrow with grading number of 2, 4, 8, 16, 32, 64, 128, and 256

number is less than or equal to 32 , the elastic modulus varies greatly with changes in the grading number, but when the grading number is greater than or equal to 64 , the elastic modulus almost remains unchanged with an increase in the grading number. Therefore, considering the accuracy of the elastic modulus and the amount of calculations together, the grading number of the elastic modulus of femur was determined to be 64 .

In the process of fracture healing, healing tissue typically goes through four stages: inflammatory stage, soft callus formation stage, hard callus formation stage, and remodeling stage. In the model, the coexistence of several kinds of healing tissues is ignored, and it is assumed that the healing tissue during a certain healing period can be characterized by a single and uniform material. Specifically, the healing tissue in the inflammatory stage is mainly in a liquid state, which can hardly bear force, and its elastic modulus is $10^{-12} \mathrm{GPa}$. During the soft and hard callus formation stages, cartilage and immature (woven) bone are dominant in the healing tissue, respectively. The elastic modulus of cartilage and immature bone are $10^{-2}$ and $1 \mathrm{GPa}$, respectively [35]. The healing tissue then enters the remodeling stage and will be completely restored to cortical bone, with elastic modulus of $20 \mathrm{GPa}$. Poisson's ratio is 0.167 in the inflammatory and soft callus stages, and 0.3 in the hard callus and remodeling stages. The ultimate strength of femoral cortical bone is between 60 and $150 \mathrm{MPa}$. The locking plate and screw are made of $22 \mathrm{Cr}-13 \mathrm{Ni}-5 \mathrm{Mn}$ stainless steel, which is regarded as a linear elastic material with elastic modulus of $200 \mathrm{GPa}$, Poisson's ratio of 0.3, and yield strength of $380 \mathrm{MPa}$ [36].

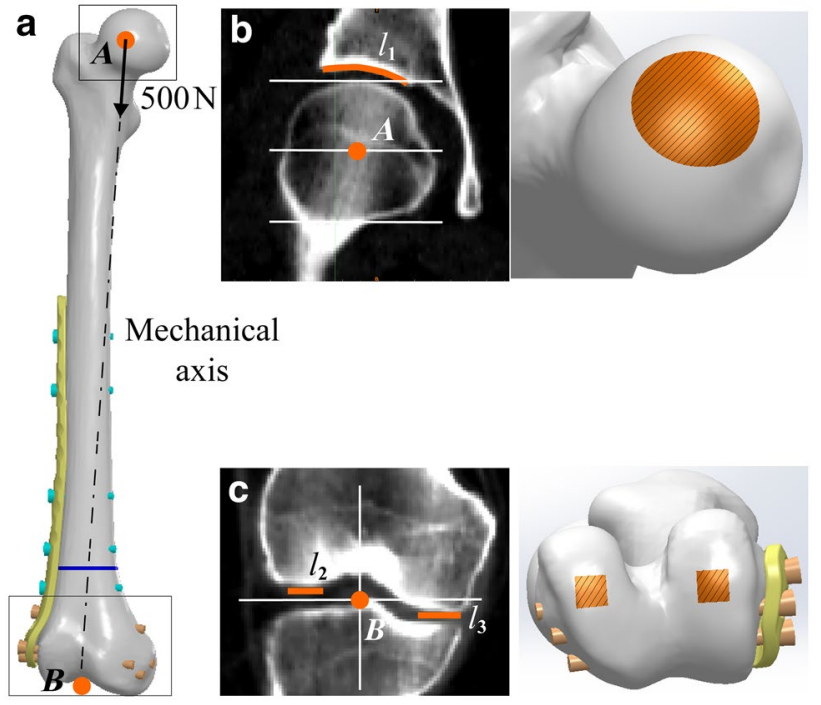

Fig. 7 Mechanical boundary of femur under single-leg standing posture. a Direction and point of applied load; $\mathbf{b}$ center of femoral head and active region; $\mathbf{c}$ center of knee joint and constraint region of supracondylar

\subsection{Boundary conditions}

The mechanical boundary of the femur is relatively simple in a single-leg standing posture, and the ligament, muscle, and joint have little effect on the stress state of the femoral condyle. Accordingly, the femur can be simplified to bear the pressure caused by human weight $(50 \mathrm{~kg})$ along the direction of its mechanical axis, that is, the central point $A$ of the femoral head to the central point $B$ of the knee joint (Fig. 7a). 

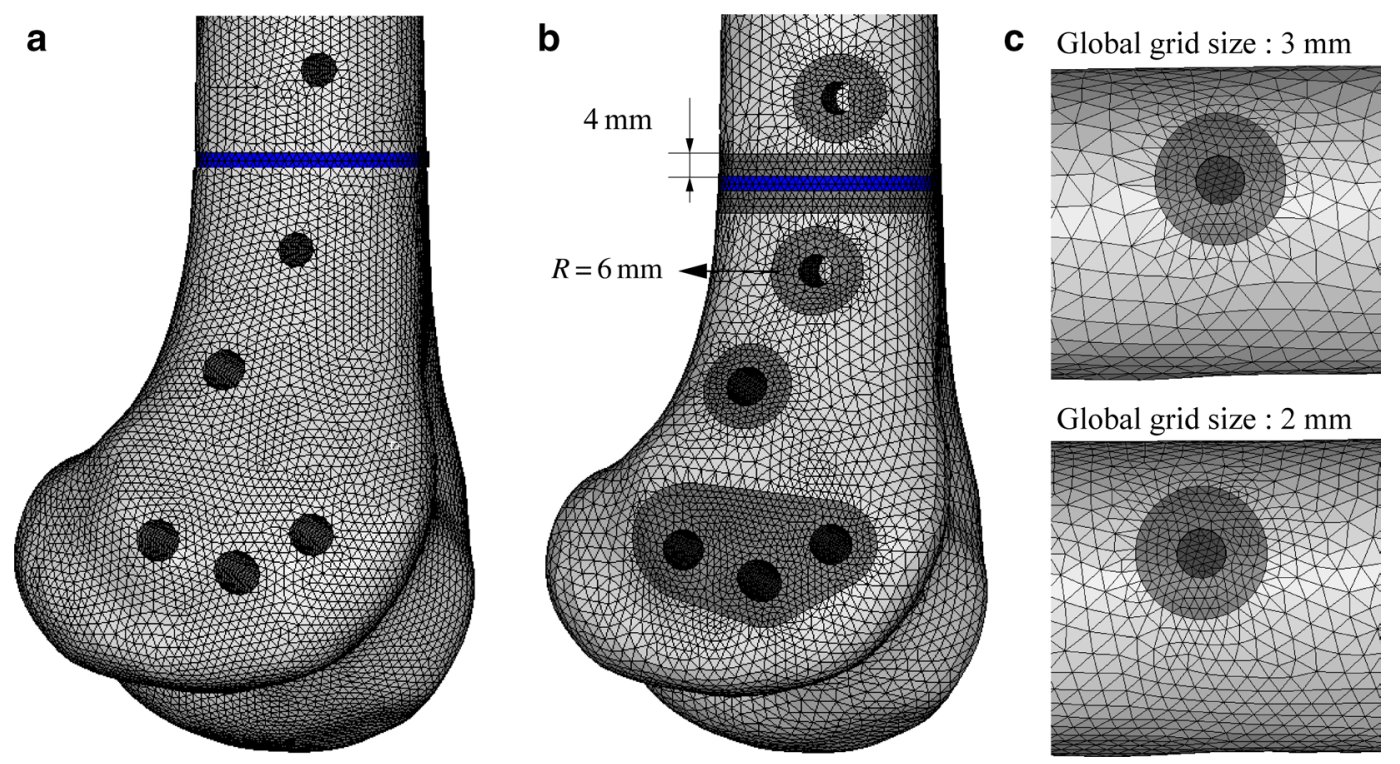

Fig. 8 Finite element model. a Overall mesh; $\mathbf{b}$ local refinement of mesh; $\mathbf{c}$ comparison between two refinement schemes in the transition area

The contact area between the femoral head and the hip is the proximal coronal area at the top of the femoral head (shaded area in Fig. 7b). The ratio of the projection of the contact area onto the cross section of the femoral head is about $1 / 3$, and the projection of the contact area onto the coronal plane is the arc $l_{1}$. By coupling the central point $A$ of the femoral head with the surface contact area of the femoral head, the resultant force of $500 \mathrm{~N}$ acting on the distributed load of the contact area is equivalent to a concentrated force applied at point $A$, and the two forces along the mechanical axis are equal in magnitude and direction.

The contact area between the femoral condyle and the tibial plateau is located on two square plateau areas of $10 \mathrm{~mm}$ $\times 10 \mathrm{~mm}$ at the bottom of the femoral condyle (shaded area in Fig. 7c). The projection of the contact area on the coronal plane is line $l_{2}$ and $l_{3}$. Given that there is no relative slip in the knee joint in the single-leg weight posture, the tibial plateau only supports the femoral condyle, so a completely fixed constraint is applied in the contact area.

The surgical fixation model of the fracture connects the two segments of the femur using screws and a steel plate. There is healing tissue between the two segments of the femur. To ensure nutritional supply to bone, there is a tiny gap of about $1 \mathrm{~mm}$ between the bone and steel plate. In the finite element model, the contact at the joint described above is simplified as follows [37-40]: the screw thread is simplified as a cylindrical surface (Fig. 1e), the screw and the steel plate are completely fixed, and a binding constraint "tie" is set up. Given that the porosity and strength of the bone are lower than those of the screw, there is likely to be slight movement between the bone and screws. Therefore, a friction contact is set up between the femoral screw hole and screw, with a friction coefficient of 0.3. When the load is larger, the femur bends and the steel plate near the fracture area might come into contact with the femur, so a friction contact is set up between them, with a friction coefficient of 0.3 . It is assumed that there is no relative movement between the femoral cross-section and the upper or lower surfaces of the healing tissue, so a binding constraint "tie" is set up.

\subsection{Mesh generation}

Hypermesh software is used to mesh the fracture fixation model, and the mesh size has a significant influence on the finite element calculation results of the femur. According to error analysis of the finite element calculation results, the upper limit of the femur mesh size is suggested to be $3 \mathrm{~mm}$ $[27,28]$. However, considering the properties of the femur element material based on QCT discrete data, the lower mesh limit is determined to be the CT spatial scanning precision of $1 \mathrm{~mm}$. In other words, when all parts of the fracture fixation model adopt the same mesh size (Fig. 8a), i.e., the overall mesh size is set to exactly $1 \mathrm{~mm}$, the total strain energy is $140.8 \mathrm{~mJ}$, and the total mesh number is 5.5 million.

Considering that the screw diameter is $4.5 \mathrm{~mm}$ and the healing tissue thickness is $3 \mathrm{~mm}$, the upper limit of the overall mesh size for the fracture fixation model is $2 \mathrm{~mm}$. When the overall mesh size is reduced from 2 to $1 \mathrm{~mm}$, the total strain energy is increased, and the mesh number increases sharply. The relative error of the total strain energy is $6.2 \%$ when the overall mesh size is $2 \mathrm{~mm}$ ( $\mathbf{\square}$ symbols in Fig. 9).

Local mesh refinement can achieve both computational accuracy and computational efficiency. The meshes are refined around the contact area between the femur and screw, 


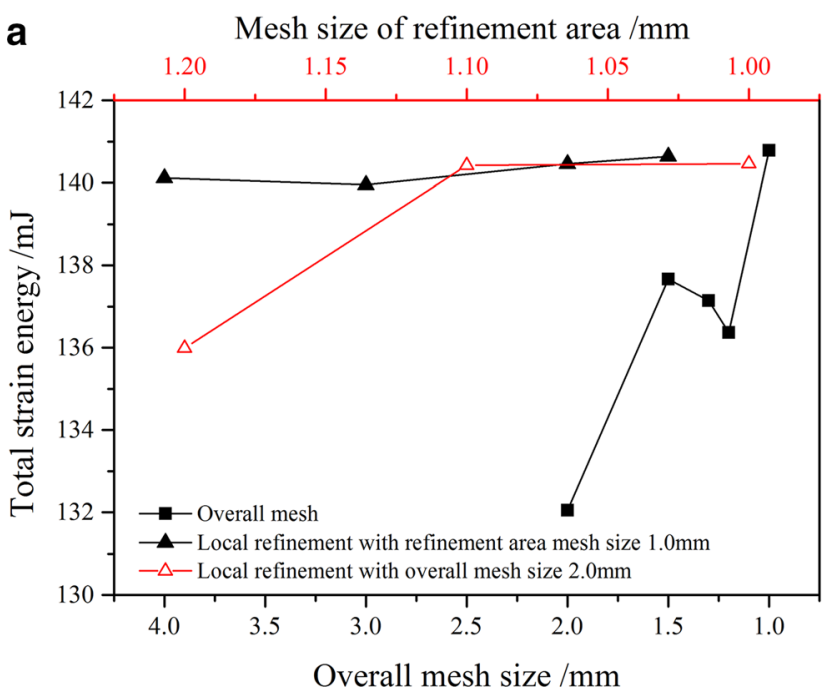

Fig. 9 Mesh convergence. a Total strain energy; $\mathbf{b}$ total mesh number

and the femur and healing tissue. The mesh size of the steel plate, screw, and healing tissue is the same as in the refinement area, while a larger overall mesh size is used in other areas of the femur. Specifically, the refinement area around the femoral screw hole is a cylindrical area with radius of $6 \mathrm{~mm}$, coaxial with the screw hole, and the refinement area around the femoral healing tissue is an area $4 \mathrm{~mm}$ from the fracture site (Fig. 8b). When the mesh size of the refinement area is $1 \mathrm{~mm}$, the overall mesh size is decreased from 4 to $1.5 \mathrm{~mm}$, and the total strain energy increases slowly ( $\Delta$ symbols in Fig. 9). Considering the quality of the element mesh in the transition area, the overall mesh size is no greater than $2 \mathrm{~mm}$ (Fig. 8c). When the overall mesh size is $2 \mathrm{~mm}$ and the mesh size of the refinement area is between $1 \mathrm{~mm}$ and $1.2 \mathrm{~mm}$, the total strain energy tends to be stable when the mesh size in the refinement area is smaller than $1.1 \mathrm{~mm}(\Delta$ symbols in Fig. 9).

Therefore, this local refinement scheme of the fracture fixation model is used in this paper. The overall mesh size and the mesh size in the refinement area are $2 \mathrm{~mm}$ and $1 \mathrm{~mm}$, respectively, and the total strain energy is $140.5 \mathrm{~mJ}$, with a relative error of only $0.2 \%$; the total mesh number is 1.5 million, which is only $27 \%$ of that when the overall mesh size is $1 \mathrm{~mm}$.

\subsection{Finite element analysis}

The meshed fracture fixation model is imported into Abaqus finite element software. Four-node tetrahedron elements are used for the mesh. Giving the material properties of elements based on QCT data, the contact setting between the femur, healing tissue, screw, and steel plate is completed,

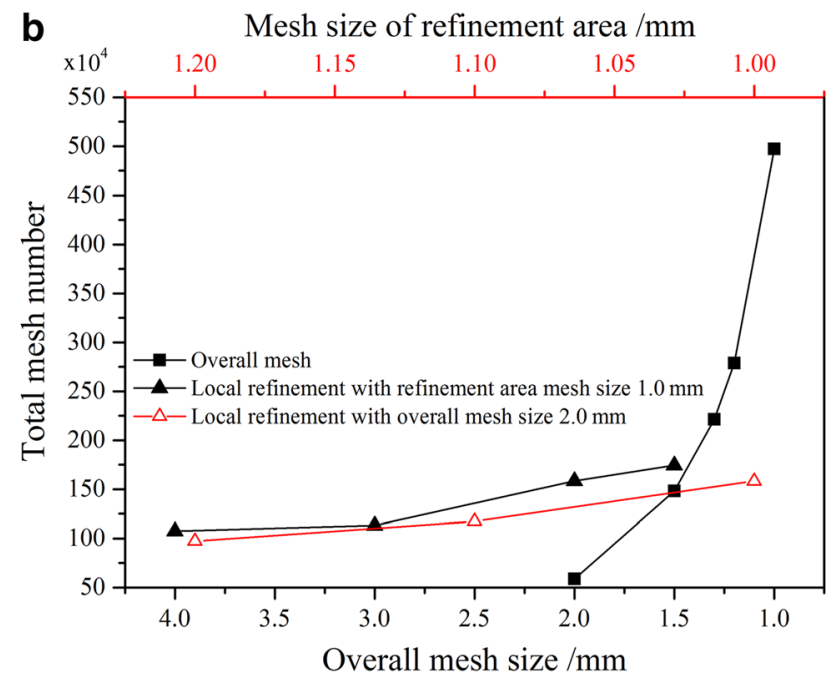

and the setting of force boundary constraints at the femoral head and femoral condyle is completed to calculate the stress distribution in the fracture fixation model in a singleleg standing posture. Figure 10 shows the stress distribution chart of fracture fixation models at different healing stages in a single-leg standing posture. From the tail end to head end of the locking plate, the screws are marked as Nos. 1-9 in turn. Figure 11 shows the stress distribution chart of an intact femur.

To analyze the stress characteristics of the fracture fixation model quantitatively, the lateral femur (on the side of the locking plate) and the medial femur (opposite to the locking plate) are obtained by dividing the femur along the anatomical axis and the sagittal plane ( $y z$ plane). The top of the femoral head is set as 0 , and the bottom of the femoral condyle is set as 1 . Along the anatomic axis, the maximum stress on any cross-section of the lateral/medial femur (Fig. 12), the maximum stress on any cross-section of the femur (including medial and lateral) (Fig. 13), and the maximum stress on the screw hole and the fracture surface of the femur (Fig. 14) are calculated. The maximum stress on each screw is counted (Fig. 15). If the $z$-coordinate of the two locking plates is nondimensionalized using the height of the 10-hole locking plate, the tail end of the 10-hole locking plate is 0 , the head end is 1 , the tail end of the 14-hole locking plate is -0.276 , and the head end is 1 ; along the $z$ axis, the maximum stress on any cross-section of the two locking plates is calculated (Fig. 16). Based on the above statistical data, Fig. 17 shows the curves of the changes in maximum stress on the femur, locking plate, and screw at different healing stages when the angles and spatial directions of the fracture face are different. 

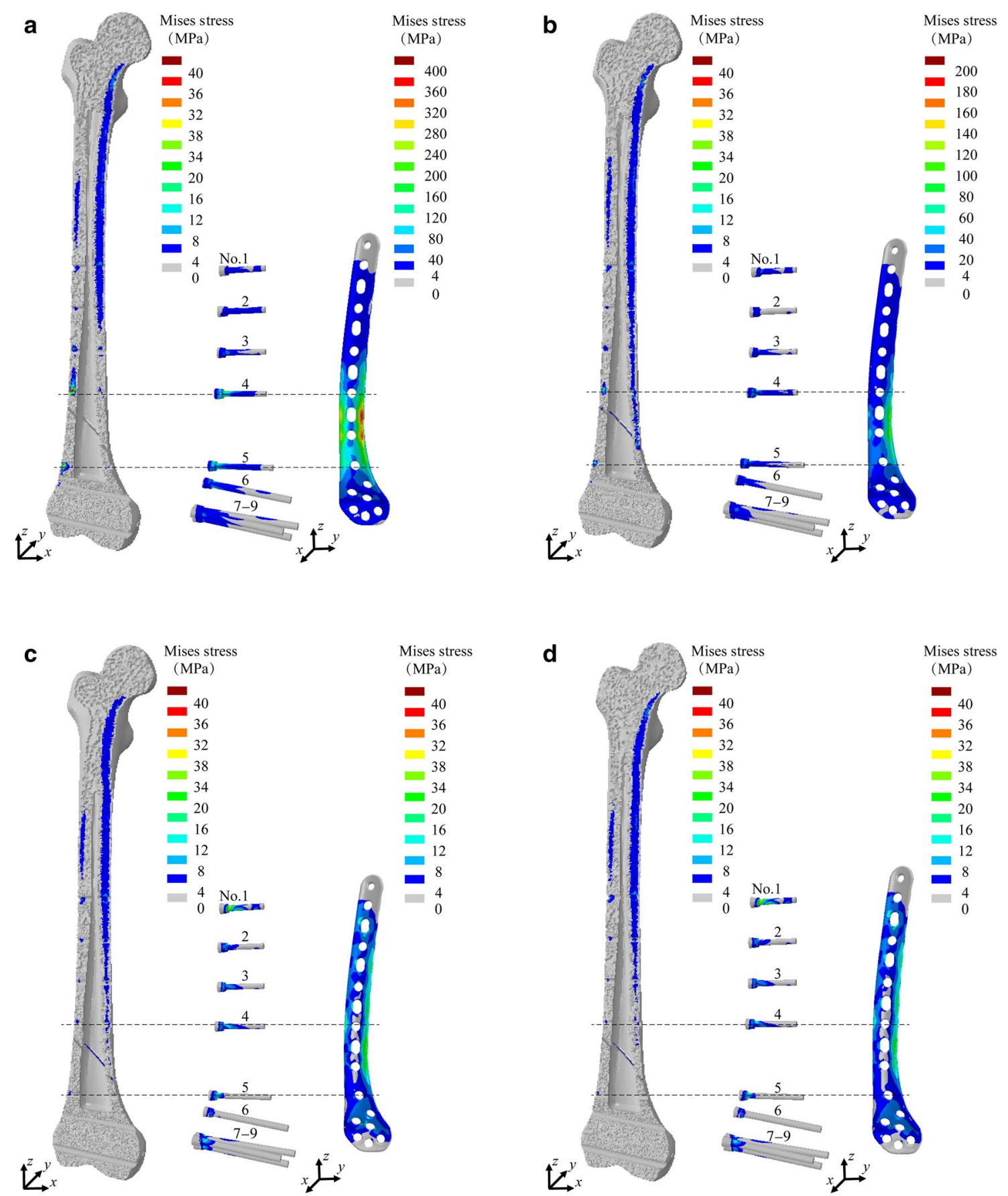

Fig. 10 Stress distribution of fracture fixation models in different healing stages in single-leg standing posture, where the callus is in the a inflammation, b soft callus formation, $\mathbf{c}$ hard callus formation, and $\mathbf{d}$ remodeling stage. The fracture angle is $45^{\circ}$, the spatial direction is I, with a 10-hole locking plate

\section{Results}

\subsection{Stress distribution characteristics}

Under the condition of $500 \mathrm{~N}$ loaded on a single leg, the stress level of the intact medial femur is slightly higher because of the asymmetry of the structure and force, being about $7 \mathrm{MPa}$, while the stress of the lateral femur is about $5 \mathrm{MPa}$ (Fig. 11). For the surgical fixation model of a simple supracondylar fracture of the femur, the stress is concentrated in the femoral screw hole, screw, and fracture site area 
Fig. 11 Stress field of intact femur in single-leg standing posture

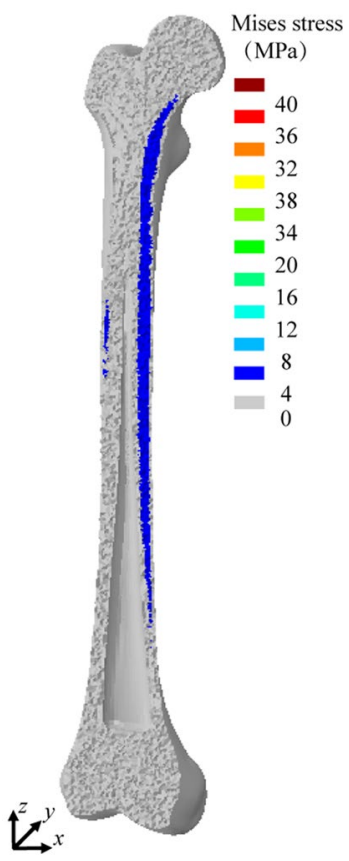

of the locking plate, and the stress concentration decreases with development of the healing tissue (Fig. 10).

In the inflammatory stage, the healing tissue is liquid. Its elastic modulus tends to 0 , so it cannot bear the load. The displacement of the fracture site is very large, ranging from 0.75 to $1.8 \mathrm{~mm}$. The main load transmission path of the fracture fixation model is medial femur-screw No. 4-locking plate-screw No. 5-lateral femur (Fig. 10a).

Firstly, the stress on the femur is concentrated at the screw hole (Figs. 12 and 14), the stress concentration is significant on screw holes Nos. 4 and 5 on both sides of the fracture site of the femur, and the maximum stress is $32.6-54.5 \mathrm{MPa}$, followed by screw hole No. 1 at the tail end of the locking plate and screw hole No. 3 adjacent to No. 4, at which the maximum stress is 12.7-20.2 MPa. The stress on the four screw holes Nos. 6-9 below the fracture surface and screw hole No. 2 in the 10-hole steel plate is lower, and the maximum stress is less than 10.0 $\mathrm{MPa}$. In addition, the stress of the lateral femur on the same screw hole is higher than that of the medial femur. In particular, when fixed with a 14-hole locking plate, the stress on screw hole No. 2 in the tail segment of the locking plate is relatively high, and the maximum stress is about $34.6 \mathrm{MPa}$, second only to that on the bilateral screw holes of the fracture site of the femur, and the stress on the medial femur is higher than that on the lateral femur. The stress distribution on the femur varies with the type of locking plate used in the fracture surface area. When fixed with a 14-hole locking plate, the maximum stress on the fracture site in the fracture surface area is $38.6-73.8 \mathrm{MPa}$ in the case of the angle of the fracture surface area of $60^{\circ}$ for direction I, III, and IV, even higher than that on the bilateral screw holes of the fracture site (Figs. 13 and 14). However, the stress on the femur is almost zero in the case of 10or 14-hole locking plate fixation (Fig. 12). Because of the stress concentration on the screw hole, for the area around the fracture site, i.e., the area from screw hole Nos. 4 and 5, the stress level of the medial/lateral femur is different from that of the intact femur, and the stress on other areas is close to that on the intact femur.

Secondly, the screw is a bridge for force transmission between the femur and locking plate. For different types of locking plate and different angles and directions of the fracture surface, the characteristics of the maximum stress distribution on each screw along the femur direction are the same. Screws Nos. 4 and 5 play the primary role in the process of force transmission. The stress of the upper screw No.
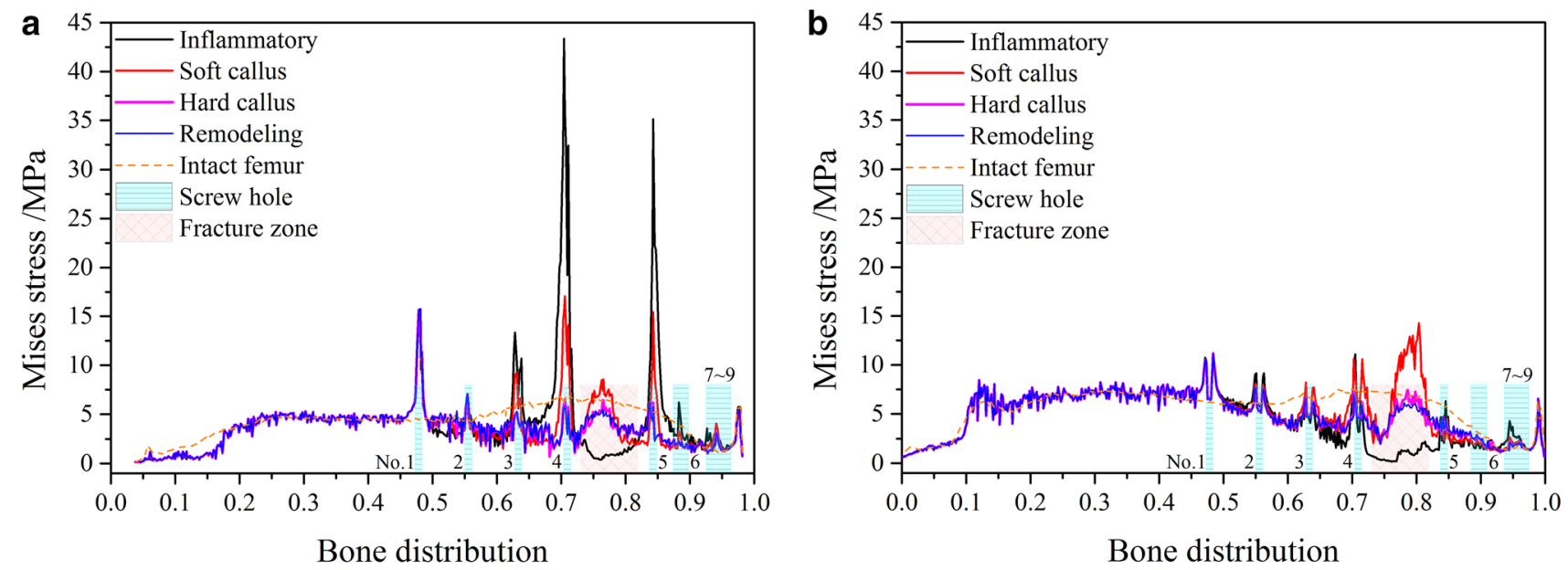

Fig. 12 Distribution curve of maximum stress along anatomic axis on any cross-section of a lateral and $\mathbf{b}$ medial femur in different healing stages. The fracture angle is $45^{\circ}$, the spatial direction is I, with a 10-hole locking plate 


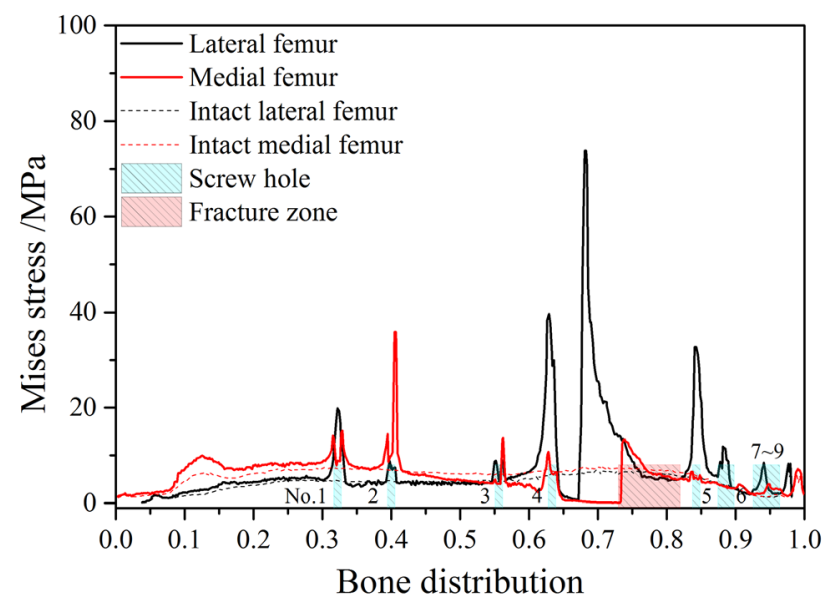

Fig. 13 Distribution curve of maximum stress on any cross-section of the femur in inflammatory stage. The fracture angle is $45^{\circ}$, the spatial direction is I, with a 10-hole locking plate

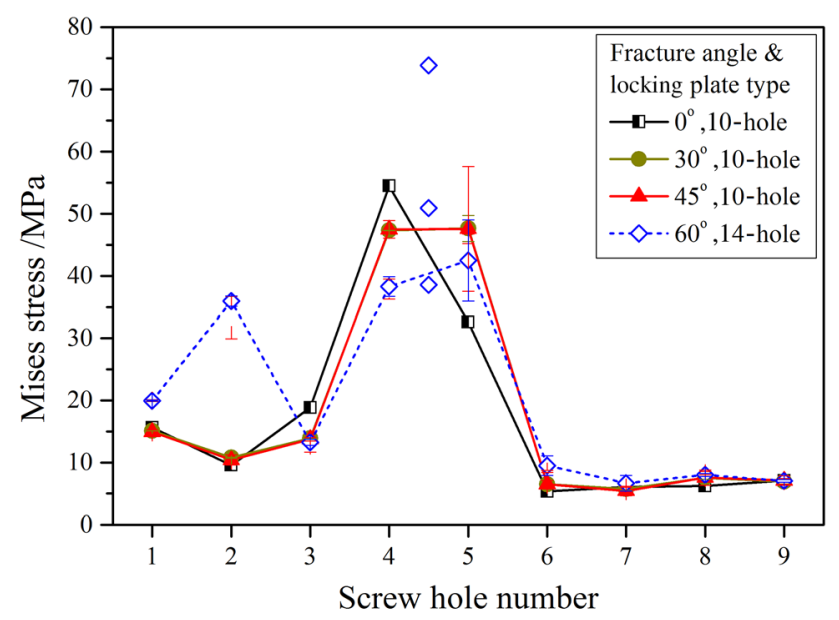

Fig. 14 Maximum stress of femur on screw hole and fracture surface in the inflammatory stage

4 is the highest, about $250-350 \mathrm{MPa}$; the stress of the lower screw No. 5 is second, and the stress of the adjacent screw No. 6 is the third; the maximum stress on the other screws is only about $50 \mathrm{MPa}$.

Thirdly, the locking plate is the main weight-bearing structure, and the axial load is transmitted along the locking plate at the fracture site. The locking plate shows obvious stress concentration between the bilateral screw holes Nos. 4 and 5 of the fracture site and its adjacent area, and the maximum stress can be up to 248-425 MPa.

The healing tissue has weak weight-bearing capacity with elastic modulus of about $10^{-2} \mathrm{GPa}$ during the soft callus stage. The displacement of the fracture site is still very large, about $16 \%-32 \%$ of that in the inflammatory stage. The main force transmission path of the fracture fixation model remains femur-screw-locking plate-screw-femur (Fig. 10b). The femoral stress on the fracture site is increased, and its maximum stress is higher than that on the intact femur. The maximum stress on the screw hole of the femur, the screw, and the fracture site area of the locking plate is significantly reduced (Fig. 17). All of the stress concentration degrees of the lateral femur on screw holes Nos. 3,4 , and 5 are decreased significantly, and the maximum stress is decreased to $39 \%-69 \%$ of that in the inflammatory stage. However, the stress on screw hole No. 1 and the other screw holes in the tail end of the locking plate in the lateral femur as well as all the screw holes in the medial femur remains almost unchanged (Fig. 12). The stresses on bilateral screws Nos. 4 and 5 of the fracture site of the femur and its adjacent screws Nos. 3 and 6 are decreased significantly, and the maximum stress is only $13 \%-37 \%$ of that in the inflammatory stage (Fig. 16a). The maximum stress on the locking plate is decreased significantly to only $24 \%-40 \%$ of that in the inflammatory stage (Fig. 17).

In the hard callus and remodeling stages, the healing tissue is differentiated into immature bone and cortical bone, and gradually recovers its weight-bearing capacity (Fig. 10c, d). The displacement of the fracture site is $0.03-0.05 \mathrm{~mm}$, which is almost equal to that of the femoral shaft. The stress concentration on the medial/lateral femur of the bilateral screw holes Nos. 4 and 5 of the fracture site and its adjacent screw hole No. 3 is further alleviated, and the stress on the fracture area tends to be consistent with that on the intact femur. However, the maximum stress on the other screw holes, especially screw hole No. 1 at the tail end of the steel plate, remains almost unchanged (Fig. 12). The stress on all screws is almost equal, only about $30 \mathrm{MPa}$ (Fig. 15a). The stress on the steel plate tends to be uniform, about $10 \mathrm{MPa}$ (Fig. 17).

It can be seen that the stress on the femur and fixation device is the highest in the inflammatory stage and is the most likely to fail. Based on the failure of the femur/fixation device, the ultimate weight load of the supracondylar fracture model of the femur with a single-leg loaded under various situations is analyzed. The ultimate strength of femoral cortical bone is $60-150 \mathrm{MPa}$ [17], and the yield strength of the steel plate and screw is $380 \mathrm{MPa}$ [29].

\subsection{Single-leg weight limit}

In the inflammatory stage, when the single-leg weight is $500 \mathrm{~N}$, the surgical fixation model of a simple supracondylar fracture of the femur with fracture surface angle of $0^{\circ} / 30^{\circ} / 45^{\circ} / 60^{\circ}$ and fracture surface directions I/II/III/IV for 10/14-hole locking plate fixation is studied. It is found that, in most cases, the maximum stress on the femur is not more than $55 \mathrm{MPa}$, and the local contact between the fracture site and locking plate results in a slightly higher stress 

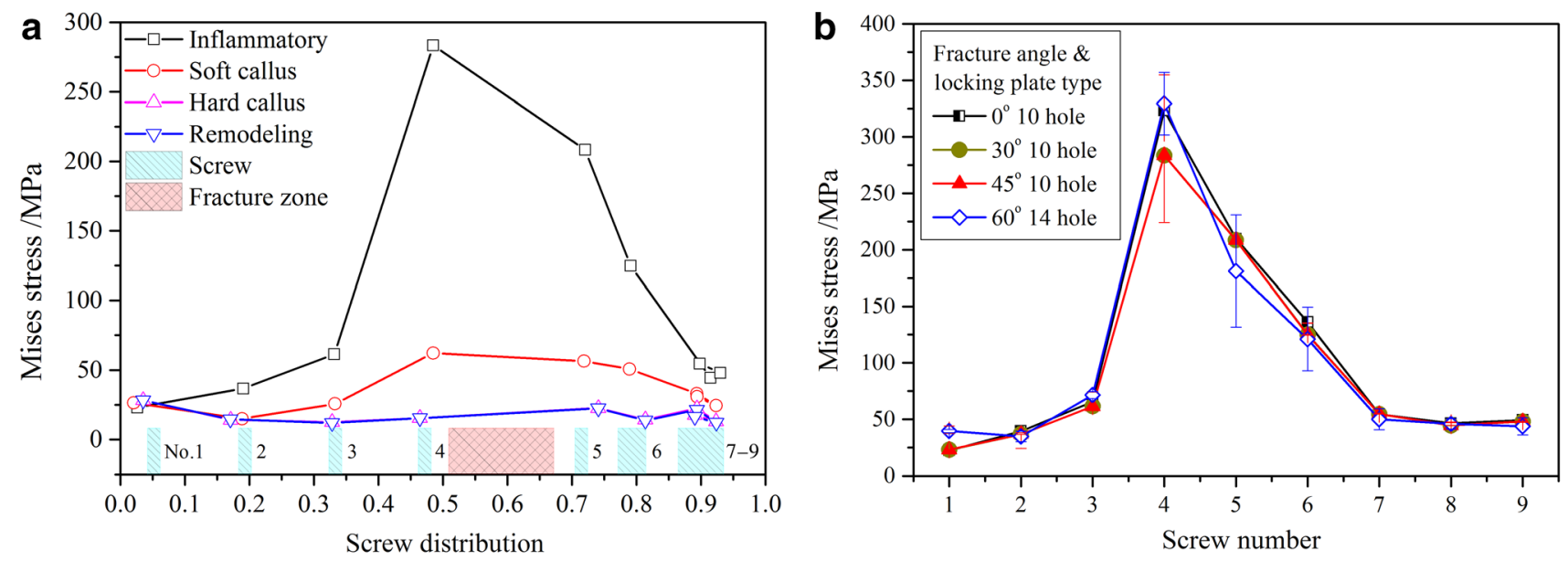

Fig. 15 Maximum stress of screw. a Fracture angle, fracture direction, and locking plate are $60^{\circ}$, I, and 14-hole, respectively. b Inflammatory stage

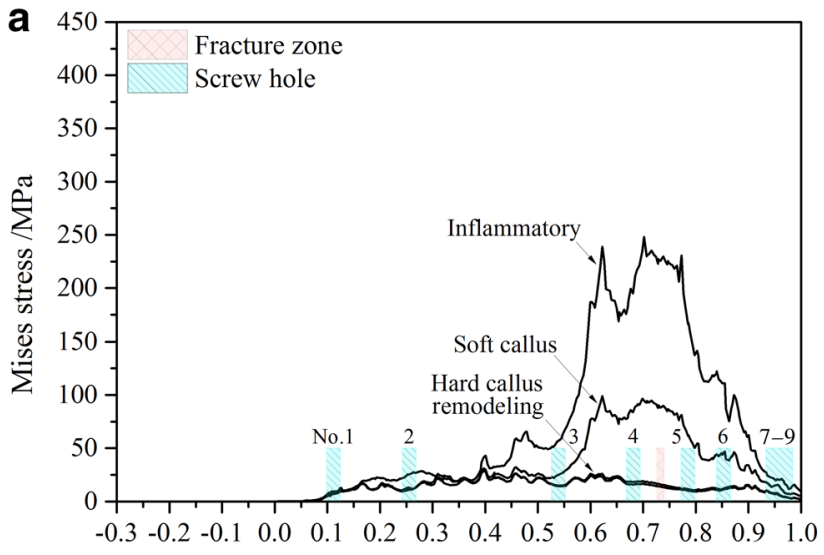

Plate distribution

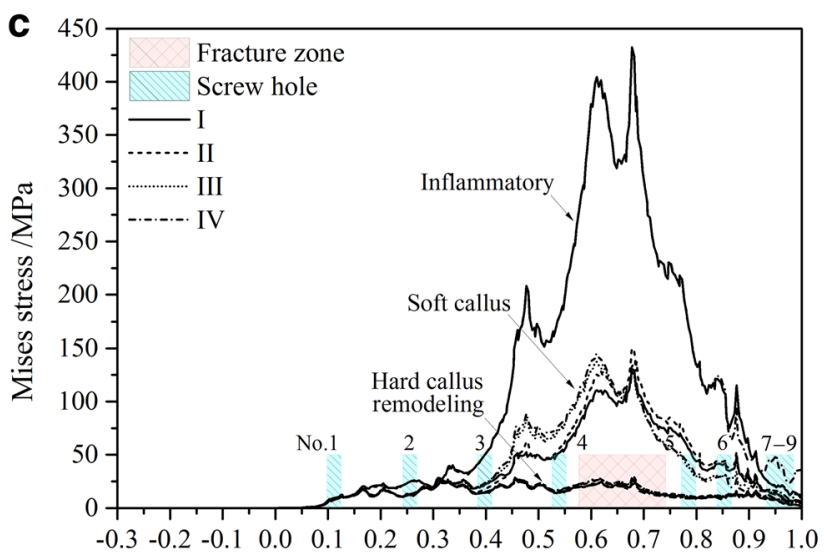

Plate distribution

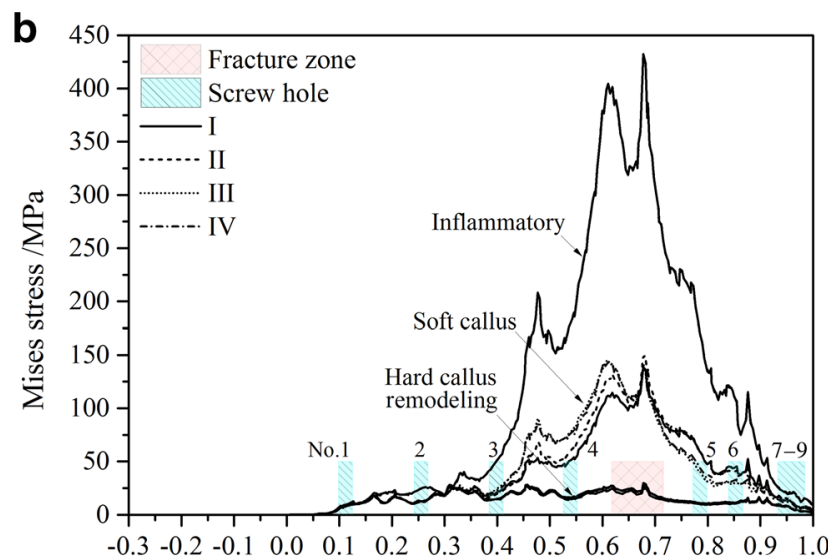

Plate distribution

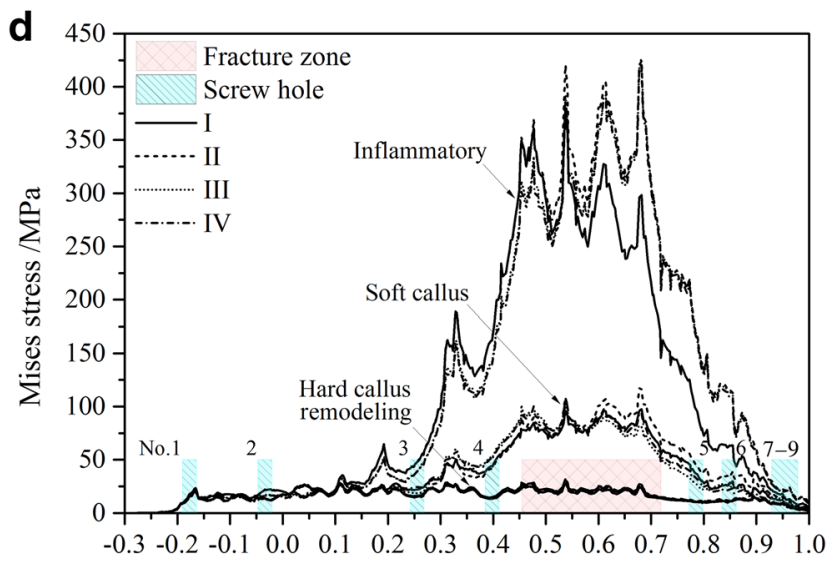

Plate distribution

Fig. 16 Stress distribution on locking plate for different healing periods and directions of fracture surface with angle of fracture and locking plate of $\mathbf{a} 0^{\circ}$ and 10 -hole, $\mathbf{b} 30^{\circ}$ and 10-hole, c $45^{\circ}$ and 10-hole, and $\mathbf{d ~} 60^{\circ}$ and 14-hole 

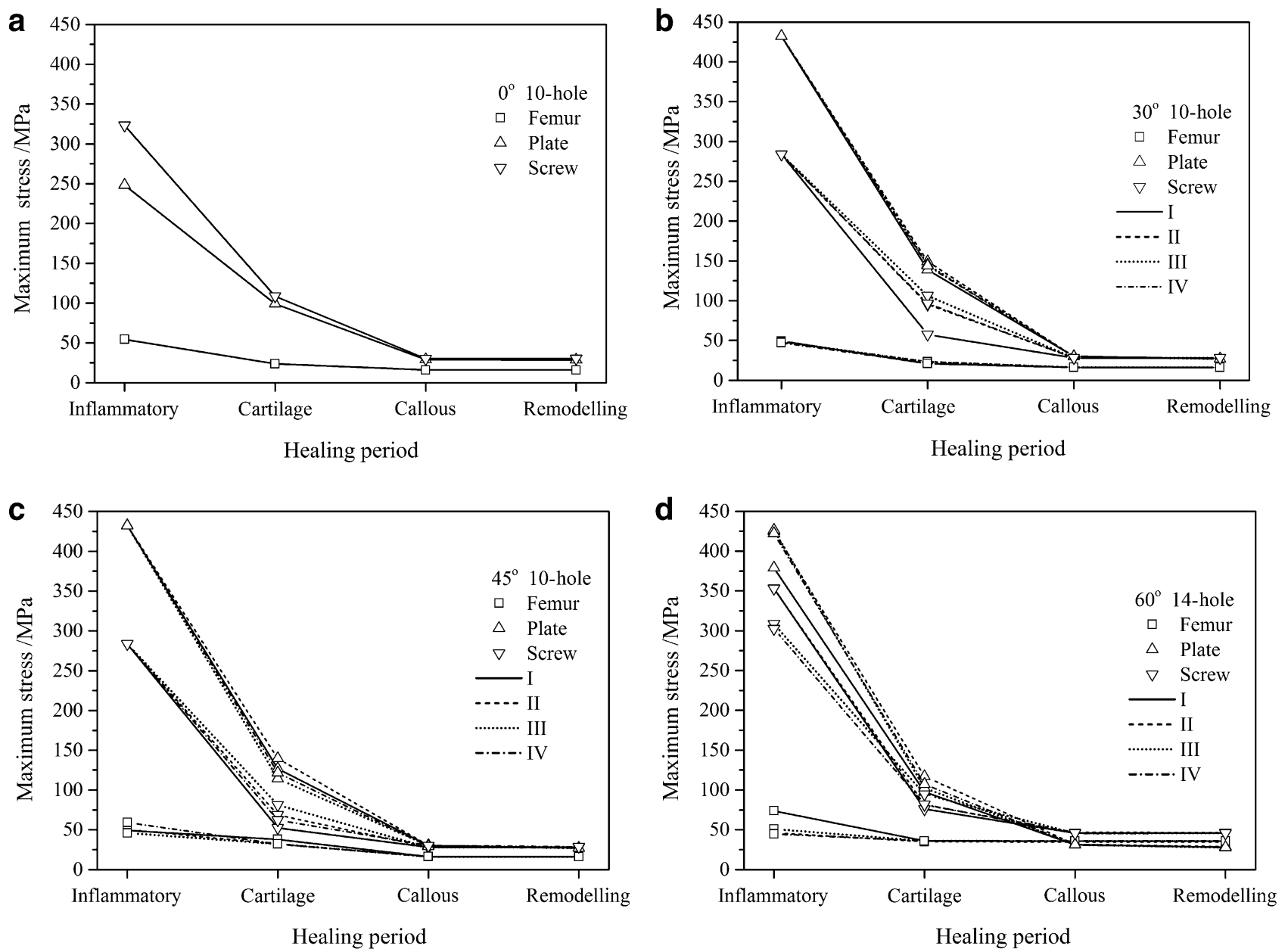

Fig. 17 Maximum stresses on locking plate, screw, and bone for different healing periods and directions of fracture surface, with angle of fracture and locking plate of $\mathbf{a} 0^{\circ}$ and 10-hole, $\mathbf{b ~} 30^{\circ}$ and 10-hole, $\mathbf{c} 45^{\circ}$ and 10-hole, and $\mathbf{d ~} 60^{\circ}$ and 14-hole

of $73.8 \mathrm{MPa}$ only for the fracture surface angle of $60^{\circ}$ with direction I and 14-hole locking plate fixation. Therefore, it can be concluded that $500 \mathrm{~N}$ weight on a single leg in the inflammatory stage will not lead to femoral failure.

For the transverse fracture fixation model, the stress on the locking plate is lower than that on the screw, and the maximum stress on the fixation device is $323.3 \mathrm{MPa}$ (Fig. 17a). For the oblique fracture fixation model with different fracture surface angles, directions, and locking plate type, the stress on the locking plate is always higher than that on the screw, and the maximum stress on the locking plate is similar for all types, about $432.3 \mathrm{MPa}$. The weight limits of the transverse and oblique fracture models are $587.7 \mathrm{~N}$ and $439.5 \mathrm{~N}$, respectively, based on fixation device failure. In other words, the weight limit of the oblique fracture model is $25 \%$ lower than that of the transverse fracture model in a single-leg standing posture.

For fracture surface angles of $30^{\circ}$ or $45^{\circ}$ and a 10-hole locking plate with different fracture surface directions, the maximum stress on the locking plate and screw is almost equal to $432.3 \mathrm{MPa}$ (Fig. 17b, c). However, the maximum stress on the fixation device is different for different fracture surface directions when the 14-hole steel plate is used. For fracture surface angle of $60^{\circ}$ and a 14-hole steel plate and fracture surface direction I, the minimum stress on the steel plate is $379.0 \mathrm{MPa}, 12 \%$ lower than that on the 10 -hole steel plate, and the situation for the other three directions is similar to for the 10-hole steel plate. For fracture surface directions I/ II and III/IV, the maximum screw stress is about $20 \%$ and $6 \%$ higher than for the 10-hole steel plate, respectively (Fig. 17d).

In the soft callus formation stage, the maximum stress on the femur is $20.9-38.1 \mathrm{MPa}$, which is $42 \%-78 \%$ of that in the inflammatory stage, and $500 \mathrm{~N}$ weight on the single leg will not cause failure of the femur. As in the inflammatory stage, the maximum stress on the locking plate is lower than that on the screw in the transverse fracture fixation model, while the maximum stress on the locking plate is higher than that on the screw in the oblique fracture fixation 
model. The maximum stresses on the transverse fracture and oblique fracture fixation models are 108.6 $\mathrm{MPa}$ and 114.2-149.3 MPa, respectively, and the weight limit of the fixation model at the soft callus stage is about 3.0-3.8 times that in the inflammatory stage.

\section{Conclusions}

A supracondylar fracture fixation model considering the angle and spatial direction of the fracture surface is comprehensively established, and the stress distribution and transmission mechanism of the fracture fixation model in single-leg standing posture are studied. On the one hand, at the early stage after operation, there is a significant stress concentration on the screw hole of the femur, the bilateral screws at the fracture site, and the fracture site area of the locking plate. With the development of callus, the stress concentration decreases significantly, and the weight limit of the fixation model in the soft callus stage is about 3.0-3.8 times as much as that in the inflammatory stage. On the other hand, the maximum stress on the transverse fracture fixation device is lower than that on the oblique fracture model, and the weight limit of the oblique fracture fixation model is $25 \%$ lower than that of the transverse fracture fixation model at the inflammatory stage. Therefore, it is necessary to consider the fracture surface angle and healing time comprehensively to determine the postoperative weight limit and improve the effect of postoperative rehabilitation.

Acknowledgements The work was supported by the National Natural Science Foundation of China (Grants 11672297, 11872273, and 11472191), the Strategic Priority Research Program of the Chinese Academy of Sciences (Grant XDB22020200), and the Opening Fund of the State Key Laboratory of Nonlinear Mechanics.

\section{References}

1. Kolmert, L., Wulff, K.: Epidemiology and treatment of distal femoral fractures in adults. Acta Orthop. Scand. 53, 957-962 (1982)

2. Richard, E.B., Christopher, G.M., Theerachai, A.: AO principles of fracture management 2 vols. (3rd edn.) Aotrauma (2017)

3. Kubiak, E.N., Fulkerson, E., Strauss, E., et al.: The evolution of locked plates. J. Bone Jt. Surg. (Am.) 88, 189-200 (2006)

4. Liang, B., Ding, Z., Shen, J., et al.: A distal femoral supra-condylar plate: biomechanical comparison with condylar plate and first clinical application for treatment of supracondylar fracture. Int. Orthop. 36, 1673-1679 (2012)

5. Macleod, A.R., Pankaj, P.: Pre-operative planning for fracture fixation using locking plates: device configuration and other considerations. Injury 49, 12-18 (2018)

6. Claes, L.: Biomechanical principles and mechanobiologic aspects of flexible and locked plating. J. Orthop. Trauma 25, 4-7 (2011)

7. Perren, S.M.: Evolution of the internal fixation of long bone fractures. The scientific basis of biological internal fixation: choosing a new balance between stability and biology. J. Bone Jt. Surg. (Bri.) 84, 1093-1100 (2002)
8. Feng, X.Q., Lee, P.V.S., Lim, C.T.: Preface: molecular, cellular, and tissue mechanobiology. Acta. Mech. Sin. 33, 219-221 (2017)

9. Adachi, T., Kameo, Y.: Computational Biomechanics of Bone Adaptation by Remodeling, vol. 578, pp. 231-259. Springer, Berlin (2018)

10. Qin, Q.H., Wang, Y.N.: A mathematical model of cortical bone remodeling at cellular level under mechanical stimulus. Acta. Mech. Sin. 28, 1678-1692 (2012)

11. Kenwright, J., Goodship, A., Evans, M., et al.: The influence of intermittent micromovement upon the healing of experimental fractures. Orthopedics 7, 481-484 (1984)

12. Goodship, A.E., Cunningham, J.L., Kenwright, J., et al.: Strain rate and timing of stimulation in mechanical modulation of fracture healing. Clin. Orthop. Relat. Res. 355, 105-115 (1998)

13. Arazi, M., Oktar, M.N., Memik, R., et al.: Early weight-bearing after statically locked reamed intramedullary nailing of comminuted femoral fractures: is it a safe procedure? J. Trauma 50, 711-716 (2001)

14. Adam, P., Bonnomet, F., Ehlinger, M.: Advantage and limitations of a minimally-invasive approach and early weight bearing in the treatment of tibial shaft fractures with locking plates. Orthop. Traumatol. Sur. 98, 564-569 (2012)

15. Kalmet, P., Sanduleanu, S., Horn, Y.V., et al.: Is early weight bearing allowed in surgically treated talar neck fractures? J. Orthop. Case Rep. 6, 73-74 (2016)

16. Elkins, J., Lawrence, M.J., Lujan, T., et al.: Motion predicts clinical callus formation: construct-specific finite element analysis of supracondylar femoral fractures. J. Bone Jt. Surg. (Am.) 98, 276-284 (2016)

17. Bottlang, M., Tsai, S., Bliven, E.K., et al.: Dynamic stabilization with active locking plates delivers faster, stronger, and more symmetric fracture-healing. J. Bone Jt. Surg. 98, 466-474 (2016)

18. Lotz, J.C., Gerhart, T.N., Hayes, W.C.: Mechanical properties of metaphyseal bone in the proximal femur. J. Biomech. 24, 317-329 (1991)

19. Lotz, J.C., Gerhart, T.N., Hayes, W.C.: Mechanical properties of trabecular bone from the proximal femur: a quantitative CT study. J. Comput. Assist. Tomogr. 14, 107-114 (1990)

20. Rho, J.Y., Hobatho, M.C., Ashman, R.B.: Relations of mechanical properties to density and CT numbers in human bone. Med. Eng. Phys. 17, 347-355 (1995)

21. Rho, J.Y., Kuhn-Spearing, L., Zioupos, P.: Mechanical properties and the hierarchical structure of bone. Med. Eng. Phys. 20, 92-102 (1998)

22. Gong, H., Zhang, M., Fan, Y., et al.: Relationships between femoral strength evaluated by nonlinear finite element analysis and BMD, material distribution and geometric morphology. Ann. Biomed. Eng. 40, 1575-1585 (2012)

23. Linwei, L., Meng, G., Gong, H., et al.: Tissue level microstructure and mechanical properties of the femoral head in the proximal femur of fracture patients. Acta. Mech. Sin. 31, 259-267 (2015)

24. Carter, D.R., Hayes, W.C.: The compressive behavior of bone as a two-phase porous structure. J. Bone Jt. Surg. (Am.) 59, 954-962 (1977)

25. Carter, D.R., Hayes, W.C.: Bone compressive strength: the influence of density and strain rate. Science 194, 1174-1176 (1976)

26. Morgan, E.F., Bayraktar, H.H., Keaveny, T.M.: Trabecular bone modulus-density relationships depend on anatomic site. J. Biomech. 36, 897-904 (2003)

27. Wirtz, D.C., Schiffers, N., Pandorf, T., et al.: Critical evaluation of known bone material properties to realize anisotropic FE-simulation of the proximal femur. J. Biomech. 33, 1325-1330 (2000)

28. Kopperdahl, D.L., Aspelund, T., Hoffmann, P.F., et al.: Assessment of incident spine and hip fractures in women and men using finite element analysis of CT scans. J. Bone Miner. Res. 29, 570580 (2014) 
29. Keyak, J.H., Skinner, H.B.: Three-dimensional finite element modelling of bone: effects of element size. J. Biomed. Eng. 14, 483-489 (1992)

30. Bessho, M., Ohnishi, I., Matsuyama, J., et al.: Prediction of strength and strain of the proximal femur by a CT-based finite element method. J. Biomech. 40, 1745-1753 (2007)

31. Garcia, J.M., Doblare, M., Seral, B., et al.: Three-dimensional finite element analysis of several internal and external pelvis fixations. J. Biomech. Eng. 122, 516-522 (2000)

32. Keyak, J.H., Rossi, S.A., Jones, K.A., et al.: Prediction of femoral fracture load using automated finite element modeling. J. Biomech. 31, 125-133 (1998)

33. Dalstra, M., Huiskes, R., Erning, L.V.: Development and validation of a three-dimensional finite element model of the pelvic bone. J. Biomech. Eng. 117, 272-278 (1995)

34. Perillo-Marcone, A., Alonso-Vazquez, A., Taylor, M.: Assessment of the effect of mesh density on the material property discretisation within QCT based FE models: a practical example using the implanted proximal tibia. Comput. Methods Biomech. 6, 17-26 (2003)

35. Isaksson, H., Wilson, W., Donkelaar, C.C., et al.: Comparison of biophysical stimuli for mechano-regulation of tissue differentiation during fracture healing. J. Biomech. 39, 1507-1516 (2006)

36. ASTM F1314-2007.: Standard specification for wrought nitrogen strengthened 22 chromium-13 nickel-5 manganese- 2.5 molybdenum stainless steel alloy bar and wire for surgical implants (2007)

37. Döbele, S., Horn, C., Eichhorn, S., et al.: The dynamic locking screw (DLS) can increase interfragmentary motion on the near cortex of locked plating constructs by reducing the axial stiffness. Langenbeck. Arch. Surg. 395, 421-428 (2010)

38. Chantarapanich, N., Sitthiseripratip, K., Mahaisavariya, B., et al.: Biomechanical performance of retrograde nail for supracondylar fractures stabilization. Med. Biol. Eng. Comput. 54, 939-952 (2016)

39. Mehboob, H., Kim, J., Mehboob, A., et al.: How post-operative rehabilitation exercises influence the healing process of radial bone shaft fractures fixed by a composite bone plate. Compos. Struct. 159, 307-315 (2017)

40. Miramini, S., Zhang, L.H., Richardson, M., et al.: The relationship between interfragmentary movement and cell differentiation in early fracture healing under locking plate fixation. Aust. Phys. Eng. S. 39, 123-133 (2016) 